\title{
INVESTIGATION OF SYNERGICIANTI-SYNERGIC INTERACTIONS OF DIHYDROXIFUMARIC ACID AND ASCORBIC ACID WITH DPPH
}

\author{
CRINA VICOL ${ }^{a *}$, CLAUDIA CIMPOIU $^{b}$, GHEORGHE DUCA ${ }^{a}$
}

\begin{abstract}
Reaction of the free-radical 2,2-diphenyl-1-picrylhydrazyl (DPPH) with dihydroxyfumaric acid (DHF) and ascorbic acid (AA) was investigated in ethanolic media and in wine simulated matrix by using a stopped-flow system. The antioxidant activity for both acids were significantly higher in wine matrix, the observed constants (kobs) being about $3 \mathrm{~s}^{-1}$ for DHF and $0.66 \mathrm{~s}^{-1}$ for AA. Synergic and anti-synergic interactions of DHF and AA against DPPH were also investigated. A strong anti-synergic effect was noticed in ethanol ( $k_{\text {obs }}<$ $\left.0.35 \mathrm{~s}^{-1}\right)$. In wine simulated matrix the constants were higher than in ethanol $\left(\mathrm{k}_{\mathrm{obs}} \approx 1 \mathrm{~s}^{-1}\right)$, but still too small for a synergic effect. Some explanations concerning the mechanisms of antioxidant action are proposed.
\end{abstract}

Keywords: dihydroxyfumaric acid, ascorbic acid, DPPH, wine matrix, stopped-flow

\section{INTRODUCTION}

Dihydroxyfumaric acid (DHF) is a 2-hydroxydicarboxylic acid consisting of fumaric acid having two hydroxy groups at the 2- and 3-positions. Fenton [1] for the first time obtained this compound in 1894 through the oxidation of tartaric acid in the presence of the hydrogen peroxide and Fe (II) [1, 2]. At that time he noticed the reduction power of DHF as long as it could reduce the $\mathrm{Ag}, \mathrm{Cu}, \mathrm{Hg}$ and permanganate salts. DHF is also a very important component in the biosynthesis of the saccharides, uronic acids and vitamin $C$ [3]. Eschenmoser [4] hypothesized that DHF along with the dihydroxymaleic and hydroxyoxalic acids represents the building blocks for the formation of most complex molecules by successive hydrolysis of hydrocyanic acid. The same idea was

\footnotetext{
a Institute of Chemistry, 3 Academiei, MD-2028, Chisinau, Republic of Moldova

b Babeş-Bolyai University, Faculty of Chemistry and Chemical Engineering, 11 Arany Janos, RO-400028, Cluj-Napoca, Romania,

*Corresponding author: crina.smigon@gmail.com
} 
experimentally supported by Sagi et al. [5]. The authors demonstrated that at the room temperature the $\mathrm{Li}, \mathrm{Cs}$ and Mg salts of DHF can interact to form more complex molecules.

DHF is also important for its antioxidant properties. Because of the higher reactivity, DHF is instable in the aqueous solutions, being oxidized to the dioxosuccinic acid [5]. It is found to be present in grapes, but in small quantities, being involved in the metabolism of the fruits during the ripening period [6]. Wines contain a low concentration of DHF because of fast decomposition in the aerobic conditions [6]. Added in small quantities (1-10 $\mathrm{mM}$ ), DHF can improve the taste and smell of wines, by inhibiting the oxidation of characteristic polyphenols $[7,8]$.

DHF and ascorbic acid (AA) are both present in wines mostly by adding them during the wine-making process, as long as their contribution is known to be positive for the quality of the beverage, and respectively, to the total antioxidant capacity of wines [9]. However, not only the presence of the reducing compound is important for the total antioxidant activity of wines. Data reported showed that the species less active as reducing agents have also a significant contribution [10, 11]. For example organic acids like tartaric, malic, citric acids found in big quantities in grapes and wines can enhance the reducing power of the main antioxidants via the synergic interactions.

Many reports [10-13] describe the synergic effect between citric, malic, acetic, tartaric acids with antioxidants from grapes and wines. These results highlight the idea that despite the insignificant antioxidant activity of the organic acids alone, when combined with primary antioxidants, they protect better the main wine compounds.

The assumption of the possible interactions between wine compounds drove the attention of researchers into this area. Thereby, high diversity of studies were performed in a wine model solution, which basically consists of tartaric acid in ethanol-water mixture with an adjusted $\mathrm{pH}$ [14-16].

Bearing all this in mind, the aim of this work was to identify the influence of different media on the antioxidant activity of less studied DHF against the free radical DPPH. In addition, the synergic or anti-synergic effect of DHF with $A A$, both applied at the concentration found in wines, was evaluated.

\section{RESULTS AND DISCUSSION}

Absorption spectra of DPPH radical in $98 \%$ ethanol and in wine simulated matrix, as well as the spectra for the reduced form of DPPH are reported in Figure 1. The determined value for the molar extinction coefficient of the stock ethanolic solution of DPPH was $11363 \mathrm{M}^{-1} \mathrm{~cm}^{-1}$, which is in perfect agreement with the data previously reported by others [17-19]. 
The maximum absorption of the free radical in ethanol is different than in wine matrix. The Figure 1 clearly illustrates this distinction, whereas the absorption maximum in ethanol is found at $517 \mathrm{~nm}$, and in wine matrix at $529 \mathrm{~nm}$. This shifting is due to the changing of solvent and of $\mathrm{pH}$ as is described in the literature $[17,20,21]$.

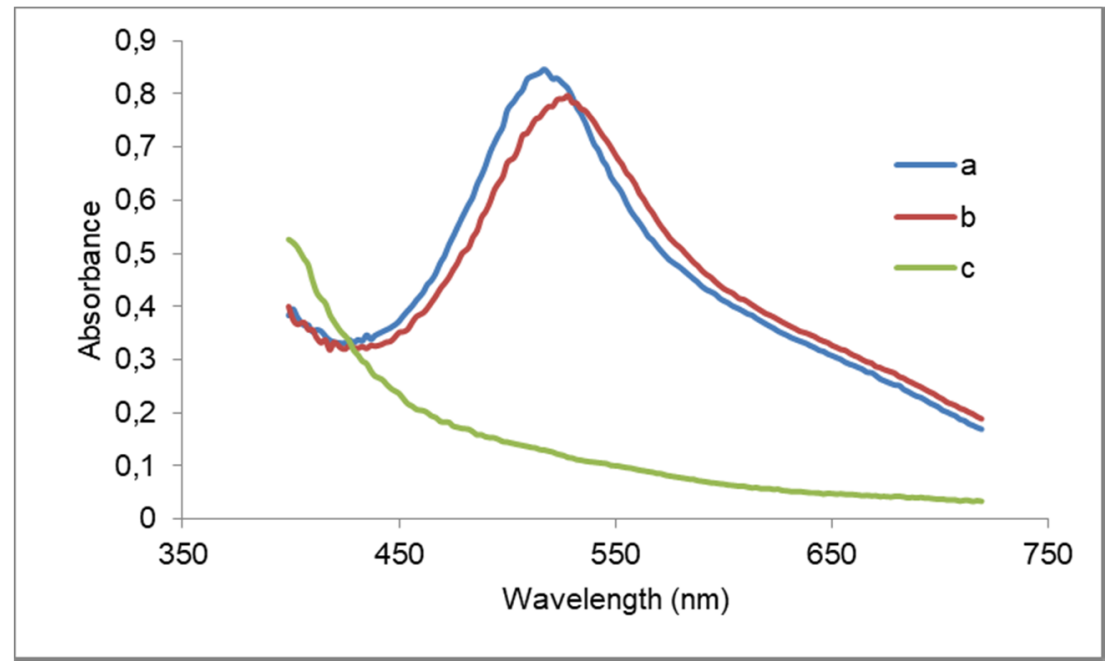

Figure 1. Absorption spectra of DPPH in ethanol (a), DPPH in wine model solution (b) and DPPH-H in wine model solution (c)

The wine simulated matrix as a whole has a small antioxidant activity against the DPPH radical. As depicted in the Figure 2, after the interaction of the DPPH with the wine matrix the decrease in absorbance is minor, the efficiency being of about 3\%. Taking into consideration the high concentration of the main constituent of the matrix - tartaric acid, the low DPPH radical scavenging activity of this compound can be underlined. This is in agreement with results obtained by others $[10,11]$.

A set of spectra were recorder after mixing the solutions of DPPH $(0.25 \mathrm{mM})$ with either $98 \%$ ethanol or wine matrix with DHF and AA at different concentrations as displayed in Figure 3. The reaction time was of 2 s. It was found that in ethanol DHF has a low antioxidant activity (Figure 3a), the maximum efficiency being of $16 \%$ for the DHF/DPPH molar ratio of 3.2 . Contrary to that, in the wine matrix the antioxidant activity of DHF is much higher. The smallest concentration of the acid in the matrix $(0.06 \mathrm{mM})$ reveals the same efficiency (16\%) as $0.8 \mathrm{mM}$ ethanolic DHF. The highest concentration of DHF reduces the free radical up to $80 \%$ (Figure $3 \mathrm{c}$ ). 


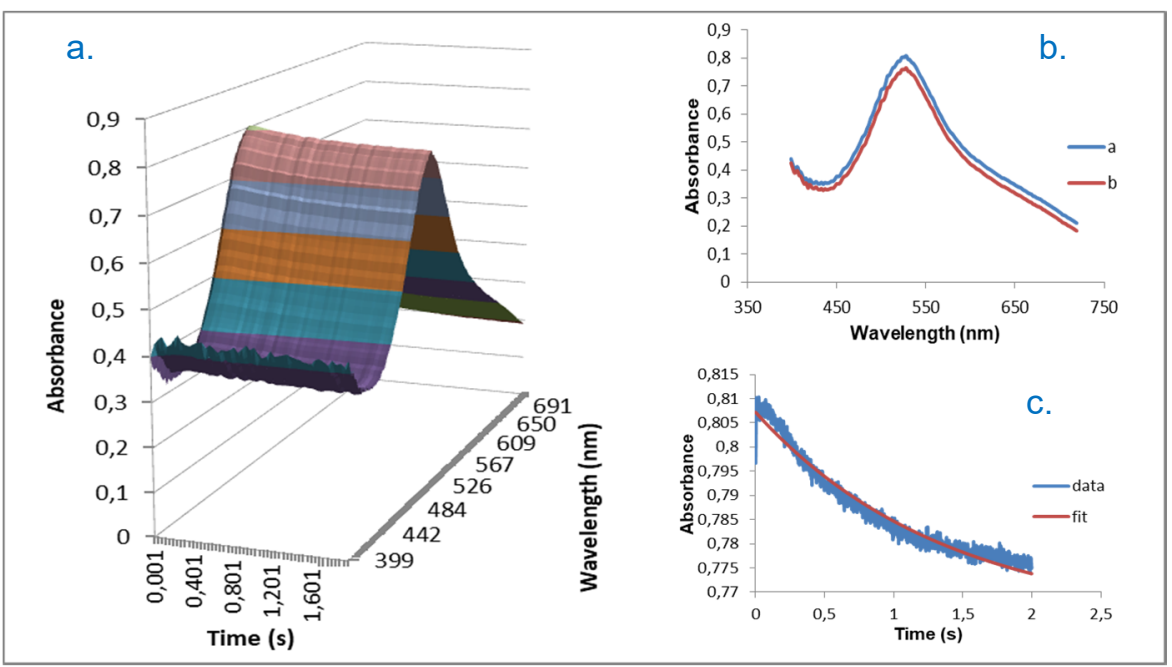

Figure 2. (a) Computed spectra of the interaction of DPPH with the wine simulated matrix. (b) Absorption spectra for the reaction of DPPH with wine simulated matrix at the time $0.001 \mathrm{sec}(\mathrm{a})$ and $2 \mathrm{sec}$ (b). (c) Fitting at the $529 \mathrm{~nm}$ for the $\mathrm{A} \rightarrow \mathrm{B}$ kinetic model
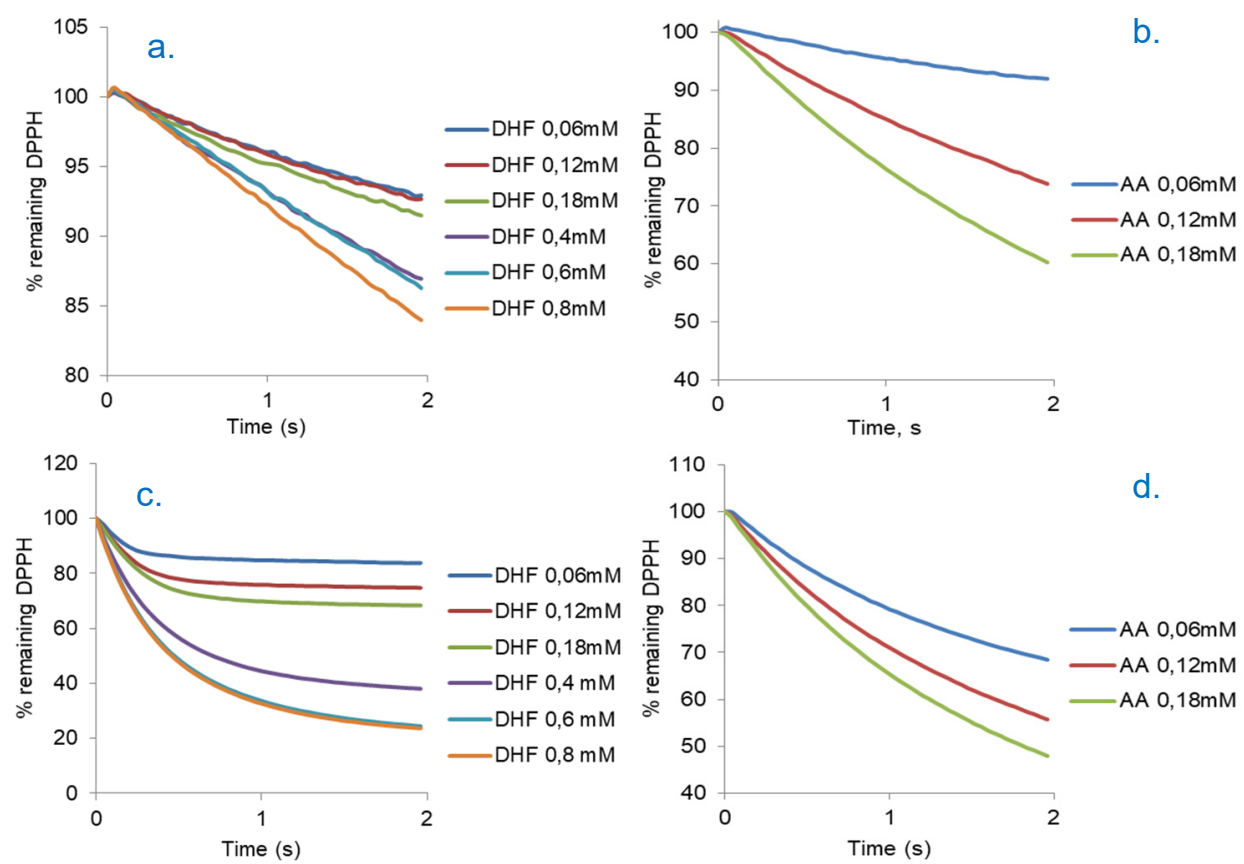

Figure 3. Spectra of the interaction of DPPH with different concentrations of DHF and $A A$ in (a), (b) ethanol, and in (c), (d) wine model solution 
As illustrated in Figure $3 \mathrm{~b}$, AA has higher antioxidant activity in ethanol comparing to that of DHF in the same solvent, since at the concentration of $0.18 \mathrm{mM} \mathrm{AA}$ has the capacity to scavenge $40 \%$ of DPPH. The same concentration of AA in the wine matrix reduces more than $50 \%$ of the free radical. A considerable change in the antioxidant activity is registered for the smallest concentration of $A A(0.06 \mathrm{mM})$, whose efficiency raises from $10 \%$ in ethanol to $30 \%$ in wine matrix (Figure $3 d$ ). Thus, the DHF show an antioxidant activity almost twice higher than that of $A A$ in both ethanol and wine model solution.

These results are supported by the observed rate constants $\left(\mathrm{k}_{\mathrm{obs}}\right)$ reported in the Table 1. As it can be noticed, in the wine matrix the $k_{\text {obs }}$ for the reactions of DPPH with DHF increase 10 times, while the constants for the reactions with $A A$ are twice higher. A similar antioxidant activity of DHF was described by Secara [20]. The author used a methanol - water solution to test the scavenging activity of DHF against DPPH, and obtained a stoichiometric constant of 2.08 for completed reaction. The solvent effect was also reported by Friaa et al. [17], once an important acceleration of the reaction was observed when ethanol-water mixtures were used instead of absolute ethanol, in favor of the electron transfer process.

Table 1. The observed rate constants in ethanol and wine model solution

\begin{tabular}{|c|c|c|c|}
\hline Sample & Concentration, $\mathrm{mM}$ & \multicolumn{2}{|c|}{$\mathrm{k}_{\text {obs, }} \mathrm{s}^{-1}$} \\
\cline { 3 - 4 } & & Ethanol & Wine model solution \\
\hline \multirow{4}{*}{ DHF } & 0.06 & $0.368 \pm 0.049$ & $3.656 \pm 0.496$ \\
\cline { 2 - 4 } & 0.12 & $0.350 \pm 0.030$ & $3.863 \pm 0.155$ \\
\cline { 2 - 4 } & 0.18 & $0.383 \pm 0.054$ & $3.371 \pm 0.091$ \\
\cline { 2 - 4 } & 0.40 & $0.167 \pm 0.024$ & $2.277 \pm 0,049$ \\
\cline { 2 - 4 } & 0.60 & $0.059 \pm 0.062$ & $2.122 \pm 0.032$ \\
\hline \multirow{3}{*}{ AA } & 0.80 & $0.040 \pm 0.016$ & $2.181 \pm 0.057$ \\
\cline { 2 - 4 } & 0.06 & $0.328 \pm 0.036$ & $0.654 \pm 0.021$ \\
\cline { 2 - 4 } & 0.12 & $0.327 \pm 0.017$ & $0.653 \pm 0.006$ \\
\hline
\end{tabular}

In order to determine the presence of synergic or anti-synergic interactions between DHF and $\mathrm{AA}$, a series of experiments were performed using six concentrations of DHF (from $0.06 \mathrm{mM}$ till $0.80 \mathrm{mM}$ ) mixed with each of the three concentration of AA $(0.06,0.12$ and $0.18(\mathrm{mM})$ (see Table 2). The experiments were realized in $98 \%$ ethanol and in wine matrix. 
The results show the same tendency as described for the antioxidants used alone with DPPH. In $98 \%$ ethanol all the mixtures exhibit low antioxidant activity compared to the values obtained in matrix. Figure 4 is well illustrating the influence of the solvent on the kinetics of the reactions. In both examples depicted in the figure the same concentrations of the antioxidants was taken $-0.18 \mathrm{mM}$ for DHF and AA, however in wine simulated matrix the free radical consumption is almost completed after $2 \mathrm{~s}$. For the mixtures with the highest concentrations of the antioxidants, the biggest efficiency in ethanol is of $20 \%$, while in the wine matrix the same combination of antioxidants scavenged $85 \%$ of the DPPH.
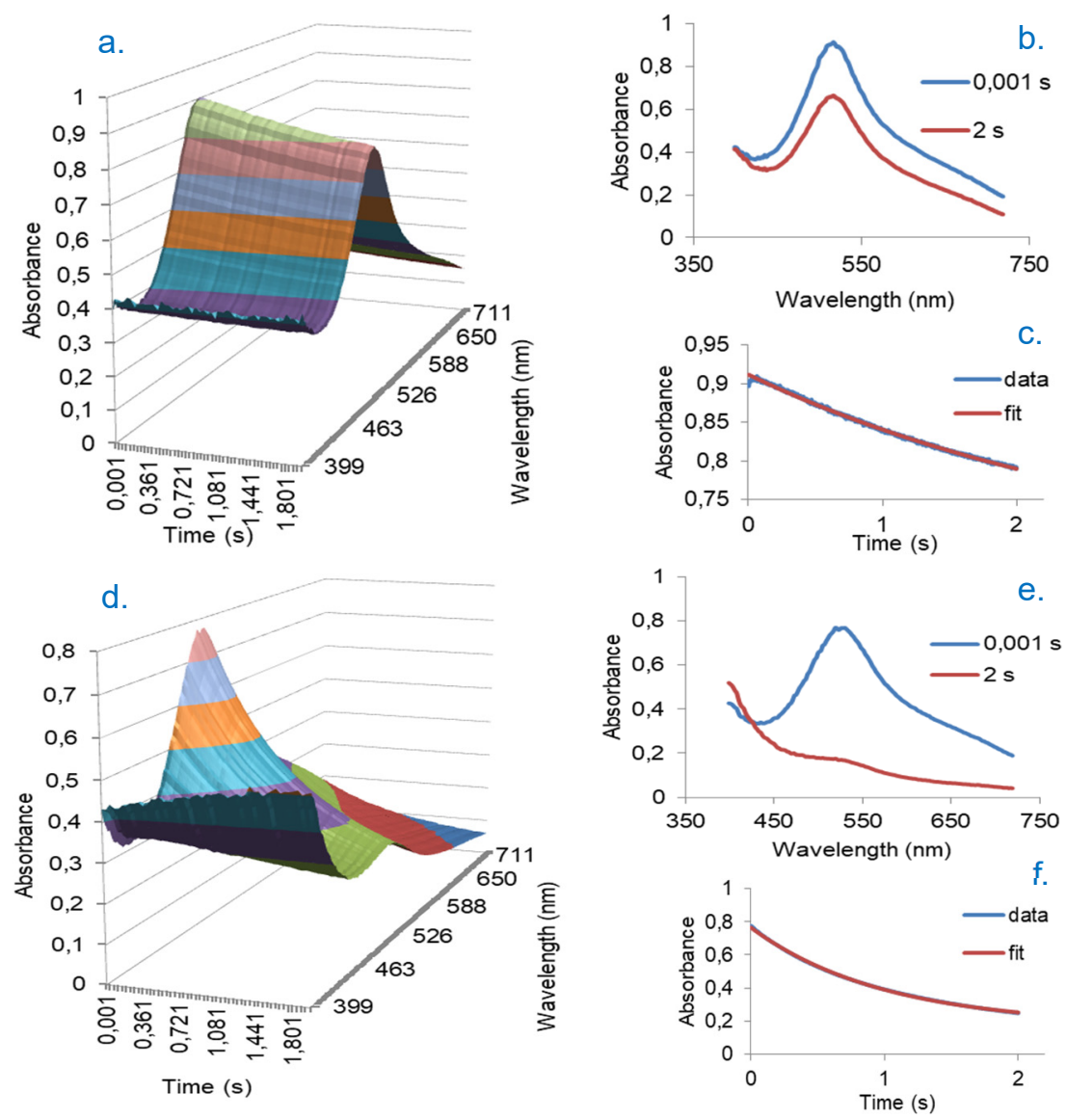

Figure 4. Decrease in absorbance after the interaction of DPPH with the mixture of DHF $(0.18 \mathrm{mM})$ and AA $(0.18 \mathrm{mM})$ in (a) $98 \%$ ethanol and (d) wine simulated matrix. Absorption spectra for the reaction of DPPH with mixture DHF - AA at the time $0.001 \mathrm{~s}$. and $2 \mathrm{~s}$. in (b) ethanol and (e) wine simulated matrix. (c), (f) Fitting at the $529 \mathrm{~nm}$ for the $\mathrm{A} \rightarrow \mathrm{B}$ kinetic model 
Data for all the combinations DHF - AA are reported in the Table 2. The values for the $\mathrm{k}_{\mathrm{obs}}$ obtained reveal a strong anti-synergism between the tested antioxidants in $98 \%$ ethanol. The biggest constant of $0.35 \mathrm{~s}^{-1}$ is registered for the mixture $\mathrm{DHF}(0.06 \mathrm{mM})-\mathrm{AA}(0,12 \mathrm{mM})$, this value being smaller than the $\mathrm{k}_{\mathrm{obs}}$ for DHF alone in ethanol (Table 1).

In the wine matrix the outcomes are improved. The constants range from $0.9 \mathrm{~s}^{-1}$ to $1.1 \mathrm{~s}^{-1}$, and are at least three time higher that the $\mathrm{k}_{\mathrm{obs}}$ in ethanol.

Despite the enhancement in the antioxidant activity of DHF - AA mixtures in the presence of the wine matrix, the anti-synergic effect still remains. This can be noticed by comparing the data in tables 1 and 2 . The observed constants for scavenging of DPPH in the wine matrix decrease from values around $3 \mathrm{~s}^{-1}$ to values around $1 \mathrm{~s}^{-1}$ when DHF is combined with $A A$ in different proportions.

Table 2. The observed rate constants in ethanol and wine model solution for the reaction of DPPH with mixtures of DHF and AA took in different concentrations

\begin{tabular}{|c|c|c|c|}
\hline \multirow{3}{*}{$\begin{array}{c}\text { Concentration } \\
\text { of AA, } \mathrm{mM}\end{array}$} & $\begin{array}{c}\text { Concentration } \\
\text { of DHF, mM }\end{array}$ & \multicolumn{2}{|c|}{$\mathrm{k}_{\text {obs, }} \mathrm{s}^{-1}$} \\
\cline { 3 - 4 } & & Ethanol & $\begin{array}{c}\text { Wine model } \\
\text { solution }\end{array}$ \\
\hline \multirow{4}{*}{0.06} & 0.06 & $0.303 \pm 0.040$ & $0.914 \pm 0.035$ \\
\cline { 2 - 4 } & 0.12 & $0.310 \pm 0.032$ & $0.962 \pm 0.026$ \\
\cline { 2 - 4 } & 0.18 & $0.311 \pm 0.021$ & $1.029 \pm 0.048$ \\
\cline { 2 - 4 } & 0.40 & $0.184 \pm 0.015$ & $1.174 \pm 0.130$ \\
\cline { 2 - 4 } & 0.60 & $0.117 \pm 0.008$ & $1.159 \pm 0.134$ \\
\hline \multirow{4}{*}{0.12} & 0.80 & $0.047 \pm 0.011$ & $1.103 \pm 0.092$ \\
\cline { 2 - 4 } & 0.06 & $0.354 \pm 0.010$ & $0.734 \pm 0.043$ \\
\cline { 2 - 4 } & 0.12 & $0.347 \pm 0.022$ & $0.873 \pm 0.014$ \\
\cline { 2 - 4 } & 0.18 & $0.322 \pm 0.035$ & $0.950 \pm 0.029$ \\
\cline { 2 - 4 } & 0.40 & $0.004 \pm 0.000$ & $0.997 \pm 0.009$ \\
\cline { 2 - 4 } & 0.60 & $0.130 \pm 0.007$ & $0.882 \pm 0.021$ \\
\hline \multirow{5}{*}{0.18} & 0.80 & $0.048 \pm 0.008$ & $0.984 \pm 0.028$ \\
\cline { 2 - 4 } & 0.06 & $0.347 \pm 0.016$ & $0.874 \pm 0.014$ \\
\cline { 2 - 4 } & 0.12 & $0.326 \pm 0.009$ & $0.946 \pm 0.008$ \\
\cline { 2 - 4 } & 0.18 & $0.333 \pm 0.017$ & $1.009 \pm 0.009$ \\
\cline { 2 - 4 } & 0.40 & $0.199 \pm 0.018$ & $1.034 \pm 0.039$ \\
\cline { 2 - 4 } & 0.60 & $0.131 \pm 0.011$ & $1.044 \pm 0.021$ \\
\hline
\end{tabular}


The literature data describes the synergic and anti-synergic effects as being dependent on the nature and concentration of the mixed compounds [22]. It is hypothesized that a combination of two or more antioxidants is considered to be synergetic as one compound can regenerate another [23]. Many authors [24-26] describes as synergetic the combinations of a weak antioxidant with a strong antioxidant, the weaker antioxidant being able to regenerate the strong antioxidant, thus improving overall radical quenching ability of the combination. Contrary to this, the anti-synergic effect assumes that the weak antioxidant is regenerated by the strongest one.

In the present experiment, the large values for the observed constants in the wine matrix can be determined by the presence of tartaric acid as a weak antioxidant that has the capacity to regenerate $A A$ and DHF when the latter ones are used alone against DPPH.

On the contrary, the anti-synergism between these two compounds may be due to the fact that $A A$ is a strong antioxidant, but has to regenerate DHF - at least in the situations when DHF is at higher concentrations. This fact can explain the decrease of the $\mathrm{k}_{\mathrm{obs}}$ for the mixture of AA with DHF $(0.40$ $-0.80(\mathrm{mM}))$.

\section{CONCLUSIONS}

The DPPH method was suitable for determining the antioxidant activity of the less studied compound (DHF) in combination with ascorbic acid in the wine matrix.

Through the stopped flow spectroscopy it was possible to study the fast reactions between DPPH and DHF and AA in 98\% ethanol and in wine simulated matrix and to compare the results.

It was found that in the wine matrix the observable constants for the single antioxidants are significantly higher than in ethanol. In the wine matrix, the $k_{\text {obs }}$ for DHF are about five times higher than the $k_{\text {obs }}$ for AA due to the synergic effect of tartaric acid.

The combination of DHF and AA exhibits a strong anti-synergism in ethanol. Although the antioxidant activity in the simulated wine matrix is higher, the anti-synergic effect remains.

\section{EXPERIMENTAL SECTION}

Ethanol (98\%) was purchased from Nordic Chemials. 2,2-Diphenyl1-picrylhydrazyl (DPPH), ascorbic acid (>99\%), DHF hydrate $(98 \%)$ and tartaric acid $(99 \%)$ were obtained from Sigma-Aldrich. 
The wine matrix was prepared by dissolving $5 \mathrm{~g} / \mathrm{L}$ of tartaric acid in a water - ethanol mixture $(12 \% \mathrm{v} / \mathrm{v})$, then the $\mathrm{pH}$ was set at 3.2 by adding sodium hydroxide [16]. The water used for the matrix was ultrapure.

Stock solutions of antioxidants were prepared twice a day, both, in $98 \%$ ethanol and in wine matrix. The $0.25 \mathrm{mM}$ DPPH solution was prepared daily only in $98 \%$ ethanol. The solutions were sonicated for 5 minutes to increase the rate of dissolution of the antioxidants and the free radical. The same procedure was done in wine simulated matrix for DHF and AA. The working wavelength was chosen based on the absorption spectrum.

UV-Vis spectra were recorded on Cary 50 UV-Vis spectrophotometer (Varian, Inc., Foster City, CA USA), using $1 \mathrm{~cm}$ quartz cuvettes. Stopped-flow spectra were collected at room temperature on a Biologic SFM-300 system equipped with three syringes and capable of sequential mixing, with a highspeed diode array detector. All experiments were done in triplicate.

Stopped-flow data were analyzed with SPECFIT32 software package (BioLogic Science Instruments, Seyssinet-Pariset, France) using Singular Value Decomposition (SVD) and global multi-exponential fitting of the SVD treated data, and the spectra were adapted to simple kinetic models using Levenberg Marquardt or Simplex Algorithms.

\section{ACKNOWLEDGMENTS}

The research was carried out within the framework of the Moldavia national project nr. 20.80009.5007.27 and with the financial support of The World Federation of Scientists and Erasmus+ Programme.

Special thanks to PhD student Maria Lehene for her guidance and support in completing the experimental part of the research.

\section{REFERENCES}

1. H.J.H. Fenton; J. Chem. Soc. Trans., 1894, 65, 899-910.

2. A.C. Clark; Eur. Food Res. Technol., 2008, 226, 925-931.

3. L. Hough; J.K.N. Jones; Nature, 1951, 167, 180-183.

4. A. Eschenmoser; Chem. Biodivers., 2007, 4, 554-573.

5. V. Naidu Sagi; P. Karri; F. Hu; R. Krishnamurthy; Angew. Chemie, 2011, 123, 8277-8280.

6. N. Secara; G. Duca; L. Vlad; F. Macaev; Chem. J. Mold., 2011, 6, 29-44.

7. B. García; R. Ruiz; J.M. Leal; J. Phys. Chem., 2008, 112, 4921-4928. 
8. L. Vacarciuc; Agricultura, 2008, 57, 1-8.

9. G. Badea; A. Antoce; Sci. Pap. B, Hortic., 2015, 59, 123-140.

10. C. Vicol; G. Duca; Rev. Şt., Inov., Cult. Art. „Akademos”, 2020, 56, 39-43.

11. R. Lo Scalzo; Food Chem.,2008, 107, 40-43.

12. W. Piang-Siong; P. De Caro; A. Marvilliers; X. Chasseray; B. Payet; A.S.C. Sing; B. Illien; Food Chem., 2017, 214, 447-452.

13. P.R. Quiroga; V. Nepote; M.T. Baumgartner; Food Chem., 2019, 277, 267-272.

14. S. Motta; M. Guaita; C. Cassino; A. Bosso; Food Chem., 2020, 313, 126045.

15. C. Dallas; J.M. Ricardo-da-Silva; O. Laureano; J. Agric. Food Chem., 1996, 44, 2402-2407.

16. P. Comuzzo; F. Battistutta; M. Vendrame; M.S. Páez; G. Luisi; R. Zironi; Food Chem., 2015, 168, 107-114.

17. O. Friaa; D. Brault; Org. Biomol. Chem., 2006, 4, 2417-2423.

18. J.M. Sendra; E. Sentandreu; J.L. Navarro; Eur. Food Res. Technol., 2006, 223, 615-624.

19. D. Villaño; M.S. Fernández-Pachón; M. L. Moyá; A.M. Troncoso; M. C. GarcíaParrilla; Talanta, 2007, 71, 230-235.

20. N. Secara; Chem. J. Mold., 2010, 5, 83-87.

21. M. Foti; C. Daquino; C. Geraci; J. Org. Chem, 2004, 14, 2309-2314.

22. U. Cornelli; Clin. Dermatol., 2009, 27, 175-194.

23. R. Tsao; Handbook of antioxidants for food preservation, Woodhead Publishing, 2015, pp. 335-347.

24. B.L. Freeman; D.L. Eggett; T.L. Parker; J. Food Sci., 2010, 75, 570-576.

25. M.N. Peyrat-Maillard; M.E. Cuvelier; C. Berset; J. Am. Oil Chem. Soc., 2003, 80, 1007-1012.

26. P. Pedrielli; L.H. Skibsted; J. Agric. Food Chem., 2002, 50, 7138-7144. 